\title{
Avaliação dos eventos sexuais de touros Nelore adultos em teste de libido realizado em curral
}

\section{Evaluation of sexual events of mature Nelore bulls in pen libido test}

\author{
Daniel Fábio Salvador, ${ }^{* \star}$ Venício José de Andrade, ${ }^{*}$ Luiz Altamiro Garcia Nogueira,, ${ }^{* *}$ Vicente Ribeiro do Vale Filho*
}

\begin{abstract}
Resumo
Um total de 38 touros adultos, aprovados andrologicamente, foi submetido ao teste de libido em curral, sendo avaliados os eventos: reflexo de Flehmen (1), perseguição ativa, com posicionamento (2), impulso de monta (3), tentativa de monta (4), monta abortada (5), monta abortada com perseguição (6) e monta completa (7). Após esta avaliação, os touros foram definidos em três categorias: alta libido - eventos 6 e 7; média libido - eventos 3 a 5 e baixa libido - eventos 1 e 2. Registrou-se alta percentagem $(68,42 \%)$ de animais com alta ou média libido. Durante os testes de libido, as freqüências médias dos eventos de 1 a 7 foram, respectivamente, $3,02 \pm 1,6 ; 0,44 \pm 079 ; 0,92 \pm 1,17 ; 0,68 \pm 0,98 ; 0,10 \pm 0,38 ; 0,10 \pm 0,31$ e $0,23 \pm 0,48$. Observouse que, após dois minutos e 30 segundos de teste, os touros de baixa libido efetuaram o dobro do evento 1 (reflexo de Flehmen), quando comparados aos touros de alta libido $(p>0,05)$. Observaram-se correlações negativas de média magnitude entre o evento 1 e os eventos 3,4 , respectivamente, -0,40 e -0,42. Já os eventos 3, 4, 6 e 7 apresentaram correlações de média para alta magnitude, com a classificação final da libido, respectivamente, 0,$47 ; 0,37 ; 0,44$ e 0,67 . Para os touros que reagiram ao teste a média do tempo para a primeira atitude de interesse por uma fêmea (TIS) foi de 40,88 $\pm 53,02$ segundos, com variações de 10 a 210 segundos. Já o tempo para a primeira atitude de monta (TAM) foi de 101,20 $\pm 74,03$ segundos, variando de 10 a 250 segundos. O TR, para os touros que efetuaram monta completa foi de 200,18 $\pm 107,77$ segundos, variando de 10 a 310 segundos.. Registraram-se correlações significativas entre TIS e TAM $(0,48)$ e de TR e TAM $(0,65)$. Conclui-se que 0 estudo detalhado dos eventos sexuais podem indicar importantes marcadores do comportamento sexual de touros da raça Nelore, direcionando as adaptações necessárias nos testes de libido em curral para touros zebuínos. Além disto, o teste de libido conforme aplicado foi eficiente em selecionar e privilegiar touros zebuínos com maior número de montas completas.
\end{abstract}

Palavras-chave: comportamento sexual, touros Nelore, Zebu.

\begin{abstract}
A total of 38 Nelore adult bulls, andrologicaly evaluated, were pen tested for libido, being observed the following Sexual reactions (SR): Flehmen reflex (1), active riding with mounting positioning (2), impulse of mounting (3), attempts to mount (4), aborted mounting (5), mounting attempts with new attempts (6), and full mounting (7). Bulls were classified as high libido (scores 6 and 7), medium libido (scores 3 to 5) and low libido (scores 1 and 2). It was registered high rate $(68.42 \%)$ of bulls with medium and high libido. Mean frequency for SR 1 to 7 were, respectively, $3.02 \pm 1.6 ; 0.44 \pm 0.79 ; 0.92 \pm 1.17 ; 0.68 \pm 0.98 ; 0.10 \pm 0.38 ; 0.10 \pm$ 0.31 e $0.23 \pm 0.48$. After 2 minutes and 30 seconds of libido test, bulls classified as low libido realized two times more the SR 1 , when compared to bulls classified as high libido $(p>0,05)$. Negative correlations among SR 1 and SR 3,4 of -0.40 e -0.42 , respectively, were recorded. SR 3, 4, 6 and 7 showed correlations varying from medium to high with final libido classification, respectively, 0,$47 ; 0.37 ; 0.44$ e 0.67 . Among bulls that react to the libido test, the mean reaction time for the first reaction to a female in heat (RF) was $40.88 \pm 53.02$ seconds (ranging from 10 to 210 seconds) and the mean mounting reaction time (MT) was $101.20 \pm 74.03$ seconds (ranging from 10 to 250 seconds). RT for bulls that realized full mounting was $200.18 \pm 107.77$ second, with variations from 10 to 310 seconds. Correlations of 0.48 between RF and MT and 0.65 between RT and MT were recorded $(p<0,05)$. It was concluded that the sexual events are important for make adaptations in pen libido test for zebu bulls. Furthermore, the pen libido test was efficient in selecting bulls that realized higher number of full mountings.
\end{abstract}

Keywords: sexual behavior, Nelore bulls, Zebu.

\section{Introdução}

A libido pode ser caracterizada como a espontaneidade ou avidez para montar e a habilidade de completar o serviço em uma fêmea e sua avaliação se inicia no momento em que o macho demonstra o interesse sexual (Hultnãs, 1959). Aparentemente as características ligadas ao comportamento sexual de touros têm certa influência da herança genética, principalmente para touros taurinos, nos quais a herdabilidade da capacidade de serviço foi estimada em 0,59

\footnotetext{
* Universidade Federal de Minas Gerais (UFMG)

** Fundação Centro de Ciência e Educação Superior a Distância do Estado do Rio de Janeiro (CECIERJ)

*** Universidade Federal Fluminense (UFF)
} 
(Blockey, 1978). Já para touros Nelore, estes valores de herança têm sido relatados com menor expressão, com a herdabilidade para libido variando de 0,30 a 0,34 (Quirino, 1999, Sarreiro, 2001). Porém, sabe-se também que tais características são de caráter multifatorial e recebem forte influência do meio e condicionamentos prévios, principalmente em touros zebuínos criados extensivamente (Santos, 2001, Costa e Silva, 2002).

Comparando-se os testes de libido, capacidade de serviço e tempo de reação para primeiro serviço em touros de corte jovens, Chenoweth et al. (1979) concluíram que o teste de libido apresenta vantagens por avaliar e refletir melhor o comportamento dos touros. Os autores ressaltaram ainda, o fato do teste de libido tratar de uma avaliação de menor duração (cinco minutos) e maior praticidade que o teste de capacidade de serviço.

Tem sido demonstrado que touros que apresentam altas classificações quanto a libido, capacidade de serviço e de razoáveis pontuações andrológicas, podem servir a maior número de fêmeas durante a estação de monta, possibilitando assim maior pressão de seleção (Vale Filho et al., 1994; Fonseca et al., 1997; Salvador et al., 2001), aliado à redução dos custos com os mesmos (Neville et al., 1988; Fonseca et al., 1997, Fonseca et al., 2000).

Entretanto, Costa e Silva et al. (1999) analisaram o comportamento em teste de libido de touros Nelore e Pantaneiro (Bos taurus taurus), criados extensivamente, evidenciando baixas freqüências de comportamentos copulatórios, de impulso e tentativa de montas, e de serviços completos, sugerindo associação da baixa freqüência destes comportamentos com a adaptação dos animais para menor desgaste nas montas a campo, em condições tropicais. O efeito de ano influenciou $(p<0,05)$ a maioria dos comportamentos observados, sugerindo baixa repetibilidade de tais observações em teste realizados em curral.

Também Santos (2001) e Costa e Silva (2002), analisando o comportamento sexual de touros Nelore, a pasto, observaram que o comportamento de reflexo ou impulso de monta foi o evento de maior importância para touros Bos taurus indicus, sugerindo ser este um comportamento estratégico para se testar a receptividade das fêmeas. Estes trabalhos levantam dúvidas quanto à efetividade dos atuais testes de libido em curral, para indicação dos melhores touros para monta a campo, assim como Oliveira et al. (2007), que não registraram diferenças nas avaliações de fertilidade a campo entre touros de alta e baixa libido. Apesar disto, Oliveira et al. (2007) citam que testes noturnos e com 30 minutos de observação podem ser mais efetivos que os testes de libido atualmente propostos (CBRA, 1998).

Já Salvador et al. (2003), usando um teste de libido com metodologia deferente dos anteriores, mostram que, com adaptações quanto ao tempo e características específicas do comportamento de touros zebuínos, os testes de libido em curral com touros Nelore adultos podem ser efetivos em selecionar touros com maior capacidade de cobertura e fertilidade a campo. Os autores relatam diferenças significativas entre a fertilidade a campo de touros de alta e baixa libido, com classificações andrológicas semelhantes.

Os resultados de Santos et al. (2004) também ressaltam a importância do teste de libido em curral como complementar ao exame andrológico, em virtude das baixas associações com outras características reprodutivas em touros Nelore, conforme já citado na literatura (Salvador et al., 2003, Oliveira et al., 2007).

Em resumo sabe-se que, apesar dos esforços de pesquisadores brasileiros das décadas de 1980 e 1990, como Pinto et al. (1989), Crudeli (1990), Costa e Silva (1994), Vale Filho et al. (1994) e Galvani (1998), na busca das associações entre o comportamento sexual, as avaliações andrológicas e a fertilidade de touros zebuínos, ainda existiam muitas dúvidas com relação ao potencial reprodutivo da espécie a campo. Já na última década, os trabalhos como os de Santos (2001), Costa e Silva (2002), Salvador et al. (2003), Santos et al. (2004) e Oliveira et al. (2007), sugerem novas abordagens de avaliação do comportamento sexual em zebuínos, tanto em curral, quanto a campo, baseado em estudos etológicos e detalhamento das análises de eventos comportamentais específicos.

Os objetivos deste experimento foram avaliar os eventos sexuais de touros Nelore adultos durante o testes de libido em curral, bem como estudar as associações entre estes eventos. Tais estudos podem ajudar na futura composição de uma avaliação mais adequada do comportamento sexual para touros zebuínos em curral e a campo, privilegiando a efetivação da monta completa.

\section{Material e métodos}

Os experimentos foram realizados na Fazenda Cristo, município de Miranda, estado do Mato Grosso do Sul, de propriedade da Agropecuária Nove de Ouro, situada às margens da rodovia BR-262, na região conhecida como "Serra da Bodoquena", em área de transição entre o planalto central e pantanal, tendo coordenadas geográficas aproximadas $20^{\circ}$ Sul, $57^{\circ}$ Oeste. O clima da região é descrito como quente e úmido, com período de chuvas que se estende de outubro a abril. A temperatura e a precipitação apresentam ciclos bem definidos, com precipitação anual média de $1600 \mathrm{~mm}$ e temperaturas máximas e mínimas de $32^{\circ} \mathrm{C}$ e $16^{\circ} \mathrm{C}$, respectivamente. $\mathrm{O}$ atual trabalho foi realizado no mês de dezembro, ou seja, no meio do período chuvoso de verão e com ótima disponibilidade de pasto.

Foram submetidos ao teste de libido 38 touros adultos (56 a 78 meses) andrologicamente normais, com base no esquema proposto por Vale Filho et al. (1994) e adaptado por Salvador et al. (2003), que sugerem que a libido seja avaliada por um período de cinco minutos, num lote de 20 fêmeas soltas no curral, das quais três em cio sincronizado previamente pelo protocolo Crestar $\AA$," podendo-se identificar três situações de libido: baixa = identificação olfativa e perseguição das fêmeas em cio; média = tentativa de salto ou salto abortado; alta = salto com penetração do pênis e galeio.

Conforme adotado por Salvador et al. (2003), inicialmente os touros permaneceram por aproximadamente 15 minutos, em lotes de três animais, em um curral adjacente ao local do teste, para observação das fêmeas em cio (pré-estimulação coletiva). Posteriormente, foram colocados individualmente em outro curral mais próximo por um período de cinco minutos (pré-

\footnotetext{
* Laboratório Intervet
} 
estimulação individual), e logo em seguida, colocado em teste por cinco minutos, para observação do comportamento sexual.

As anotações foram feitas por dois observadores posicionados em pontos distintos do curral, sempre escondidos para não interferirem no comportamento dos animais.

Os principais eventos comportamentais avaliados foram os adotados por Salvador (2001), que realizou um apanhado dos principais comportamentos já descritos na literatura (Vale Filho et al., 1994; Fonseca et al., 1997, Costa e Silva et al. 1999 e Santos, 2001) para touros zebuínos. Estes eventos foram: 1) Reflexo de Flehmen (RF); 2) Perseguição ativa, com posicionamento (PA); 3) Impulso ou reflexo de monta (IM); 4) Tentativa de monta (TM); 5) Monta abortada (MA); 6) Monta abortada com perseguição (MAP); 7) Monta completa (MC).

Após o término do teste de libido, foi feita a leitura complementar ou média entre as planilhas dos observadores, sendo os touros classificados em três categorias: alta libido - comportamentos 6 e 7; média libido - comportamentos 3, 4 e 5 ; baixa libido - comportamentos 1 e 2 .

Foi anotado também o horário de início do teste até os referidos comportamentos, para posterior análise. Foram calculados os intervalos de tempos para primeira atitude de interesse sexual por uma fêmea (TIS) e tempo para a realização da primeira monta completa, denominado tempo de reação (TR), como descritos por Chenoweth et al. (1979) e Costa e Silva et al. (1999), bem como o tempo para primeira descritas, onde a alta libido representou o valor três, a média libido o valor dois e a baixa libido valor um. Em todas estas análises utilizando-se dos recursos computacio-nais do pacote estatístico SAS (1996).

\section{Resultados e discussão}

Observou-se uma resposta adequada dos touros ao referido teste, uma vez que alta percentagem de animais $(68,42 \%)$ atingiram a classificação média $(39,47 \%)$ e alta libido $(28,95 \%)$. Os resultados foram similares aos relatados por Costa e Silva et al. (1999) e Oliveira et al. (2007), onde o total de animais classificados como baixa ou questionáveis libido, variaram na faixa dos $30 \%$, dependendo do tempo de aplicação do teste.

Não houve aparente inibição do comportamento dos animais, sugerindo ter sido adequada a metodologia aplicada, aliado ao fato de se ter trabalhado somente com touros adultos e com experiência sexual prévia.

Diferentemente de outros testes propostos na literatura (Chenoweth et al., 1979; Pineda et al., 1997; CBRA, 1998), este foi um teste de 25 minutos de estimulação sexual para os touros, do início ao final do exame, apesar dos touros terem apenas cinco minutos de contato direto com as fêmeas, quando os eventos sexuais foram observados.

$\mathrm{Na}$ Tabela 1 são apresentados os resultados das freqüências médias por touro, durante os testes de libido, dos comportamentos de 1 a 7 para touros de alta, média e baixa libido.

Tabela 1: Freqüências médias dos comportamentos observados durante o teste de libido em curral, para touros Nelore adultos

\begin{tabular}{|c|c|c|c|c|c|c|c|}
\hline \multirow{2}{*}{ Libido } & \multicolumn{7}{|c|}{ Comportamentos durante o teste de libido em curral } \\
\hline & $\mathrm{RF}(1)$ & POS (2) & $\mathrm{IM}(3)$ & TM (4) & MA (5) & MAP (6) & MC (7) \\
\hline Alta & $2,63 \pm 1,43$ & $0,36 \pm 0,67 a b$ & $1,18 \pm 1,16$ & $0,81 \pm 1,07$ & $0,27 \pm 0,64$ & $0,36 \pm 0,50$ & $0,81 \pm 0,60$ \\
\hline Média & $2,80 \pm 1,61$ & $0,80 \pm 1,01 \mathrm{a}$ & $1,46 \pm 1,24$ & $1,13 \pm 1,06$ & $0,06 \pm 0,25$ & - & - \\
\hline Baixa & $3,66 \pm 1,87$ & $0,08 \pm 0,28 b$ & - & - & - & - & - \\
\hline Geral & $3,02 \pm 1,66$ & $0,44 \pm 0,79$ & $0,92 \pm 1,17$ & $0,68 \pm 0,98$ & $0,10 \pm 0,38$ & $0,10 \pm 0,31$ & $0,23 \pm 0,48$ \\
\hline
\end{tabular}

OBS: Os dados se referem às médias e desvio-padrão das freqüências. A comparação foi feita com Teste de Kruskal \& Wallis, $p<0,05$. Nas lacunas não preenchidas os números são inexistentes.

atitude de monta (TAM), que no caso de touros Nelore foi considerado como sendo o impulso de monta.

As comparações de médias foram realizadas através de delineamento inteiramente casualisado. Para comparação das médias de tempo para as reações sexuais usou-se SNK, ao nível de $5 \%$. Já as comparações das freqüências dos comportamentos dos touros foram realizadas como análises não-paramétricas usando-se o teste de Wilcoxon, para pares ordenados e teste de Kruskal e Wallis para grupos diferentes de animais, segundo Sampaio (1998). Para a análise das correlações entre os eventos comportamentais foi usado o cálculo do coeficiente de correlação de Spearman, recomendado para dados não-paramétricos, também segundo Sampaio (1998). As correlações e médias para a libido foram calculadas considerando-se apenas as três categorias
Observa-se que os touros examinados quanto a libido tiveram como comportamentos mais freqüentes o reflexo de Flehmen, o impulso de monta e a tentativa de monta, mostrando a importância de tais atitudes na possibilidade de previsão do futuro comportamento de touros Nelore, como relatado por Santos (2001) e Costa e Silva (2002). Já Oliveira et al. (2007) relatam que os principais eventos fisiológicos do comportamento sexual de touros Nelore em teste de curral foram: o ato de cheirar ou lamber o corpo da fêmea, cheirar ou lamber a vulva, seguidos pelo reflexo de Flehmen.

$\mathrm{Na}$ comparação das médias registraram-se diferenças $(p<0,05)$ apenas para o evento 2 , entre os touros de baixa e média libido.

Diante da alta prevalência do comportamento de reflexo de Flehmen e sua importância dentro das atitudes de cortejo e 
verificação da receptividade das fêmeas em touros Nelore (Santos, 2001, Costa e Silva, 2002, Oliveira et al., 2007), optouse por uma análise mais detalhada deste evento (Tabela 2).

Tabela 2: Freqüência média de atitudes de reflexo de Flehmen $(\mathrm{RF})$, realizadas por touros Nelore adultos, durante os testes de libido em curral

\begin{tabular}{cccc}
\hline Libido & Até 2h30 minutos & Após 2h30 minutos & Geral \\
\hline Alta & $2,00 \pm 1,34$ & $0,63 \pm 1,02$ & $2,63 \pm 1,43$ \\
Média & $1,60 \pm 1,29$ & $1,20 \pm 0,94$ & $2,80 \pm 1,61$ \\
Baixa & $2,25 \pm 1,48$ & $1,41 \pm 1,24$ & $3,66 \pm 1,87$ \\
\hline
\end{tabular}

Os números se referem às médias e os desvio-padrão das freqüências. A comparação foi feita com Teste de Wilcoxon, $p>0,05$.

Deve-se salientar a maior freqüência do comportamento do reflexo de Flehmen nos touros de baixa libido quando comparado aos touros de alta libido, sem, contudo, atingir diferença estatística $(p>0,05)$, sugerindo ser este um comportamento mais associado à identificação e primeiro contato dos touros com as fêmeas e pouco relacionado com a monta completa, como já descrito pela literatura (Costa e Silva et al., 1999; Santos, 2001). Isto também é confirmado pelas correlações negativas do RF com IM e TM, respectivamente, $-0,40$ e $-0,42(p<0,05)$.

Verificou-se ainda, que não houve diferença $(p>0,05)$ na atitude de reflexo de Flehmen (RF), entre os touros de alta, média e baixa libido até os primeiros dois minutos e 30 segundos do teste (Tabela 2). No entanto, após este período e até aos cinco minutos (final do teste), a freqüência de atitudes de RF, observados nos touros de baixa libido, foi o dobro daquele observado nos touros de alta libido (porém ainda com p>0,05), possivelmente em virtude destes últimos já estarem manifestando os demais comportamentos relacionados com a monta.

Aparentemente, os touros de alta libido foram mais diretos ou mais rápidos na realização dos comportamentos copulatórios, com menor freqüência de RF que os touros classificados como baixa libido. Pode-se supor, também, que os touros com atitudes sexuais mais relacionados com a monta completa durante os testes de libido em curral, podem também ser os de maior dominância a campo, como descrito por Costa e Silva (1998). Neste trabalho observou-se nos touros Nelore, em acasalamentos múltiplos, o estabelecimento da relação de dominância, onde os subordinados praticavam maior freqüência de comportamentos de identificação de fêmeas no cio e os dominantes mostrando-se mais rápidos em chegar aos eventos copulatórios.

Na Tabela 3 são apresentados os coeficientes de correlação de Spearman entre os diversos comportamentos observados em teste de libido em curral, os intervalos de tempo de reação (TR), tempo para primeira atitude de monta (TAM) e tempo para primeira atitude de interesse sexual por uma fêmea (TIS) e sua classificação final quanto à libido.

Observaram-se correlações negativas de média magnitude entre o RF com IM e TM, respectivamente, -0,40 e -0,42 ( $p<0,05)$, confirmando os resultados já descritos de que touros que praticaram mais RF tiveram menor freqüência de comportamentos relacionados com os eventos copulatórios.

Foi observada uma correlação positiva entre o IM e a classificação final da libido de 0,47 $(p<0,05)$, confirmando a importância deste evento na classificação final do teste de libido. Estes resultados concordam com afirmações de Costa e Silva (2002), de que o comportamento de IM é uma atitude importante e está ligada ao início dos comportamentos de monta completa em touros Nelore. Também Salvador et al. (2003), citam o impulso de monta com um importante evento sexual, sendo o evento mais freqüente observado durante as avaliações de campo, com alto número de fêmeas em cio.

Os comportamentos de IM, TM, MAP (monta abortada com perseguição) e MC apresentaram correlações de média para alta magnitude, com a classificação final da libido, como já era esperado, por serem eles ligados a MC, que define os touros de alta libido.

Observou-se alguma associação entre TR e IM (correlação de 0,59 , porém com $p=0,05$ ), sugerindo que a freqüência deste comportamento estaria relacionado com um maior tempo para a realização do serviço completo em testes de libido em curral.

Em virtude dos altos coeficientes de variação dos períodos de tempo de reação (TR), tempo para primeira atitude de monta (TAM) e tempo para primeira atitude de interesse sexual por uma fêmea (TIS), os dados sofreram transformação logarítmica para uma análise mais adequada de suas correlações, bem como das comparações de suas médias, conforme recomendação de Sampaio (1998).

Na Tabela 4 pode-se observar que a média geral do TIS ( $\mathrm{n}=$ 34 touros), foi de 40,88 \pm 53,02 segundos, com variações de 10 a 210 segundos. Já o TAM ( $n=25$ touros) foi de 101,20 \pm 74,03 segundos, variando de 10 a 250 segundos. O TR, para os 11 touros que efetuaram monta completa foi de 200,18 \pm 107,77, variando de 10 a 310 segundos.

Quando foram comparados os tempos de TR, TAM e TIS entre os grupos de alta, média e baixa libido, observou-se que touros de alta libido apresentaram menor média de tempo para TIS e TAM, apesar de não haver diferença estatística ( $>0,05)$ por causa do alto desvio-padrão. Mesmo assim, a diferença numérica expressiva indica que os animais que efetuaram monta completa durante o teste foram mais rápidos em demonstrar interesse pelas fêmeas e iniciar os eventos relacionados com a monta.

As médias gerais de TIS e TR, para todos os touros, foram semelhantes às obtidas por Costa e Silva et al. (1999), para touros das raças Nelore e Pantaneira, porém com o desviopadrão superior, o que sugere uma maior variabilidade de reação dos touros às fêmeas, registrada no presente estudo. Porém o tempo de reação foi inferior ao relatado por Chenoweth et al. (1979), em testes de capacidade de serviço realizados em touros de corte jovens, com duração de 10 minutos.

$\mathrm{Na}$ Tabela 5 são apresentados os coeficientes de correlação simples entre TIS, TAM e TR observados durante o teste de libido em curral. Observou-se correlação significativa entre TIS e $\operatorname{TAM}(0,48)$, sugerindo que à medida que os touros desencadeiem mais rapidamente os comportamentos de identificação das fêmeas em cio (TIS), estes serão mais rápidos em iniciar os comportamentos relacionados com a monta (TAM). 
Tabela 3: Coeficientes de correlação de Spearman, entre os comportamentos, a classificação final da libido e os períodos TIS, TAM e TR, observados durante os testes de libido em curral, para touros Nelore adultos

\begin{tabular}{|c|c|c|c|c|c|c|c|}
\hline & RF & POS & IM & TM & MA & MAP & $\mathrm{MC}$ \\
\hline POS & $\begin{array}{c}0,10 \\
(0,5163)\end{array}$ & 1 & - & - & - & - & - \\
\hline IM & $\begin{array}{c}-0,40 \\
(0,0128)\end{array}$ & $\begin{array}{c}0,17 \\
(0,3043)\end{array}$ & 1 & - & - & - & - \\
\hline $\mathrm{TM}$ & $\begin{array}{c}-0,42 \\
(0,0084)\end{array}$ & $\begin{array}{c}-0,10 \\
(0,5284)\end{array}$ & $\begin{array}{c}0,31 \\
(0,0536)\end{array}$ & 1 & - & - & - \\
\hline MA & $\begin{array}{c}-0,07 \\
(0,6455)\end{array}$ & $\begin{array}{c}-0,00 \\
(0,9770)\end{array}$ & $\begin{array}{c}0,11 \\
(0,5037)\end{array}$ & $\begin{array}{c}0,20 \\
(0,2210)\end{array}$ & 1 & - & - \\
\hline MAP & $\begin{array}{c}-0,09 \\
(0,5676)\end{array}$ & $\begin{array}{c}0,15 \\
(0,3471)\end{array}$ & $\begin{array}{c}0,19 \\
(0,2492)\end{array}$ & $\begin{array}{c}0,08 \\
(0,5946)\end{array}$ & $\begin{array}{c}-0,10 \\
(0,5489)\end{array}$ & 1 & - \\
\hline$M C$ & $\begin{array}{c}-0,18 \\
(0,2555)\end{array}$ & $\begin{array}{c}-0,08 \\
(0,6023)\end{array}$ & $\begin{array}{c}-0,01 \\
(0,9425)\end{array}$ & $\begin{array}{c}0,08 \\
(0,5964)\end{array}$ & $\begin{array}{c}0,31 \\
(0,0503)\end{array}$ & $\begin{array}{c}0,02 \\
(0,8694)\end{array}$ & 1 \\
\hline LIBIDO & $\begin{array}{c}-0,31 \\
(0,0580)\end{array}$ & $\begin{array}{c}0,17 \\
(0,3011)\end{array}$ & $\begin{array}{c}0,47 \\
(0,0027)\end{array}$ & $\begin{array}{c}0,37 \\
(0,0213)\end{array}$ & $\begin{array}{c}0,26 \\
(0,1100)\end{array}$ & $\begin{array}{c}0,44 \\
(0,0047)\end{array}$ & $\begin{array}{c}0,67 \\
(0,0001\end{array}$ \\
\hline TIS & $\begin{array}{c}-0,20 \\
(0,2564)\end{array}$ & $\begin{array}{c}0,02 \\
(0,8888)\end{array}$ & $\begin{array}{c}0,01 \\
(0,9255)\end{array}$ & $\begin{array}{c}-0,01 \\
(0,9204)\end{array}$ & $\begin{array}{c}-0,03 \\
(0,8626)\end{array}$ & $\begin{array}{c}-0,16 \\
(0,3411)\end{array}$ & $\begin{array}{c}-0,08 \\
(0,6322\end{array}$ \\
\hline TAM & $\begin{array}{c}0,27 \\
(0,1821)\end{array}$ & $\begin{array}{c}0,27 \\
(0,1864)\end{array}$ & $\begin{array}{c}-0,27 \\
(0,1761)\end{array}$ & $\begin{array}{c}-0,32 \\
(0,1112)\end{array}$ & $\begin{array}{c}-0,33 \\
(0,0995)\end{array}$ & $\begin{array}{c}-0,00 \\
(0,9713)\end{array}$ & $\begin{array}{c}-0,32 \\
(0,1105\end{array}$ \\
\hline TR & $\begin{array}{c}0,28 \\
(0,4032)\end{array}$ & $\begin{array}{c}0,11 \\
(0,7464)\end{array}$ & $\begin{array}{c}0,59 \\
(0,0553)\end{array}$ & $\begin{array}{c}0,35 \\
(0,2845)\end{array}$ & $\begin{array}{c}0,38 \\
(0,2421)\end{array}$ & $\begin{array}{c}0,17 \\
(0,5970)\end{array}$ & $\begin{array}{c}-0,37 \\
(0,2572\end{array}$ \\
\hline
\end{tabular}

OBS: Entre parênteses estão os graus de significância para cada correlação apresentada.

Tabela 4: Tempos para primeira atitude de interesse sexual por uma fêmea (TIS), para primeira atitude de monta (TAM) e de reação (TR), observados durante os testes de libido em curral, para touros Nelore adultos

\begin{tabular}{cccc}
\hline Libido & TIS & TAM & TR \\
\hline Alta & $18,81 \pm 8,54^{\underline{a}}$ & $70,00 \pm 46,55$ & $200,18 \pm 107,77$ \\
Média & $50,57 \pm 57,33^{\underline{a}}$ & $125,71 \pm 83,54$ & - \\
Baixa & $52,77 \pm 71,89^{\underline{a}}$ & - & - \\
Geral & $40,88 \pm 53,02$ & $101,20 \pm 74,03$ & $200,18 \pm 107,77$
\end{tabular}

OBS: Os dados se referem às médias e desvio-padrão dos tempos. A comparação foi feita com Teste SNK, $p>0,05$. As lacunas não preenchidas são de dados inexistentes.

Tabela 5: Coeficientes de correlação simples, entre os tempos de interesse (TIS), atitude de monta (TAM) e de reação (TR)

\begin{tabular}{ccc}
\hline & TIS & TAM \\
\cline { 2 - 3 } TAM & $0,48(0,0124)$ & - \\
TR & $0,26(0,4327)$ & $0,65(0,0274)$ \\
\hline
\end{tabular}

OBS: Entre parênteses estão os graus de significância para cada correlação apresentada.
Observou-se ainda correlação significativa entre os tempos de TR e TAM $(0,65)$, indicando que touros mais rápidos em manifestar os comportamentos relacionados com a monta (principalmente o impulso de monta), efetuaram mais rapidamente a monta completa em teste de libido em curral, ressaltando a importância do impulso de monta como um evento sexual marcador do comportamento de touros Nelore que vão realizar a monta completa.

As associações entre os tempos de reação aos eventos sexuais mostram tendência de desencadeamento em cascata dos comportamentos sexuais de touros zebuínos em curral. O estudo futuro mais detalhado dos eventos sexuais e o tempo de reação para estes eventos podem indicar importantes marcadores do comportamento sexual de touros da raça Nelore, direcionando as adaptações necessárias nos testes de libido em curral para touros zebuínos.

\section{Conclusões}

Os eventos comportamentais de "reflexo de Flehmen", "impulso de monta", "tentativa de monta" foram os mais freqüentes e importantes na avaliação da libido de touros zebuínos em curral.

O "impulso de monta" e "tentativa de monta" estiveram associados à classificação final da libido, mostram-se determinantes na indicação dos touros com maiores chances de realizar monta completa em curral. 
As associações entre os tempos para primeira reação de interesse (TIS), monta (TAM) e cópula (TR), mostram uma tendência de desencadeamento em cascata dos comportamentos sexuais iniciadas pelo "impulso de monta", que foi o evento que esteve também mais associado ao tempo de reação (TR).
O teste de libido conforme aplicado foi eficiente em selecionar e privilegiar touros zebuínos com maior número de montas completas.

\section{Agradecimentos}

À Fazenda Cristo, FAPEMIG, CNPq pelo apoio logístico e financeiro.

\section{Referências}

BLOCKEY, M. A. de B. et al. Heritability of serving capacity and scrotal circumference in beef bulls. J. Anim. Sci., v. 47, suppl. 1, p. 253, 1978. COLÉGIO BRASILEITO DE REPRODUÇÃO ANIMAL-CBRA. Manual para exame andrológico e avaliação de sêmen animal. 2. ed. Belo Horizonte: CBRA, 1998. 49 p.

CHENOWETH, P. J.; BRINKS, J. S.; NETT, T. M. A comparison of the three methods of sex-drive in yearling beef bulls and relationships with testoterone and LH levels. Theriogenology, v. 12, n. 4, p. 223233, 1979 .

COSTA E SILVA, V. E. Avaliação da capacidade reprodutiva de touros Nelore. Exame andrológico, teste de comportamento sexual e desafio da fertilidade. (Dissertação de Mestrado). Belo Horizonte: Escola de Veterinária, UFMG, 1994.102p.

COSTA ESILVA, E. V.; SERENO, J. R. B.; ANDRIOLO, A. etal. Freqüência de montas por touros Nelore (Bos taurus indicus) em sistema de acasalamento múltiplo: efeito da hierarquia. In: ENCONTRO ANUAL DE ETOLOGIA, 16., 1998, São José do Rio Preto. Anais... São José do Rio Preto: SBEt, p. 48, 1998.

COSTA E SILVA, V. E.; SERENO, J. R. B.; PARANHOS DA COSTA, M. J. R. et al. Comportamento sexual de touros Nelore (Bos taurus indicus) e pantaneiro (Bos taurus taurus) durante os procedimentos de teste de libido. Rev. Bras. Reprod. Anim. v. 23, n. 3, p. 214-216, 1999.

COSTA E SILVA, E. V. Comportamento sexual de touros Nelore (Bos taurus indicus) em monta a campo e em testes de libido. (Tese de Doutorado em Zootecnia). Jaboticabal: Faculdade de Ciências Agrárias e Veterinárias, Universidade Estadual Paulista, 2002. 137 p.

CRUDELI, G. A. Avaliação da aptidão reprodutiva de touros de raça Nelore e seu efeito sobre a taxa de gestação do rebanho. (Dissertação de Mestrado). Belo Horizonte: Escola de Veterinária, UFMG, 1990, 135 p.

FONSECA, V. O.; FRANCO, C. S.; BERGMANN, J. A. G. et al. Potencial reprodutivo de touros da raça Nelore (Bos taurus indicus) acasalados com elevado número de vacas. Arq. Bras. Med. Vet. Zootec., v. 49, n. 1, p. 53-62, 1997.

FONSECA, V. O.; FRANCO, C. S.; BERGMANN, J. A. G. Potencial reprodutivo e econômico de touros Nelore acasalados coletivamente na proporção de um touro para 80 vacas. Arq. Bras. Med. Vet. Zootec., v. 52, n. 1, p. 77-82, 2000.

HULTNÃS, C. A. Studies on variation on mating behavior and semen picture in young bulls of the swedish red-and-white bred and on causes of this variations. Acta Agric. Scand., v. 9, n. 6, p. 1-89, 1959. GALVANI, F. Desempenho reprodutivo de touros de alta libido da raça Nelore. Viçosa, MG: Universidade Federal de Viçosa, 1998. 69 p. Dissertação (Mestrado em Medicina Veterinária) - Universidade Federal de Viçosa, 1998.
NEVILLE, Jr., W. E.; WILLIAMS, D. J.; RICHARDSON, K. L. et al. Relationship of breeding soundness evaluation score and it's components with reproductive performance of beef bulls. Theriogenology, v. 30, n. 3, p. 429-436, 1988.

OLIVEIRA, C. B.; GUIMARÃES, J.D., COSTA, E.P et al. Avaliação do comportamento sexual em touros Nelore: comparação entre os testes da libido em curral e do comportamento sexual a campo. Rev. Bras. Zootec., v. 36, n. 1, p. 32-42, 2007.

PINEDA, N. R.; LEMOS, P. F.; FONSECA, V. O. Comparação entre dois testes de avaliação do comportamento sexual (libido) de touros Nelore (Bos taurus indicus). Rev. Bras. Reprod. Anim., v. 21, n. 4, p. 29-34, 1997.

PINTO, P. A.; SILVA, P. R.; ALBUQUERQUE, L. G. Avaliação da biometria testicular e a capacidade de monta em bovinos das raças Guzerá e Nelore. Rev. Bras. Reprod. Anim., v. 13, n. 3, p. 151-156, 1989.

QUIRINO, C. R. Herdabilidades e correlações genéticas entre medições testiculares, características seminais e libido em touros Nelore. (Tese de Doutorado). Belo Horizonte: Escola de Veterinária, UFMG, 1999. 78 p.

SALVADOR, D. F.; ANDRADE, V. J.; VALE FILHO, V. R. etal. Desempenho reprodutivo de touros da raça Nelore, submetidos à classificação andrológica por pontos (CAP), à libido e desafiados com alto número de fêmeas com estro sincronizado. Rev. Bras. Reprod. Anim., v. 25, n. 2, p. 185-187, 2001.

SALVADOR, D. F.; ANDRADE, V.J.; VALE FILHO, V. R. et al. Avaliação da libido de touros Nelore adultos em curral e sua associação com características andrológicas e desempenho reprodutivo a campo. Arq. Brás. Med. Vet. Zootec., v. 55, n. 5, p. 588-593, 2003.

SAMPAIO, I. B. M. Estatística aplicada à experimentação animal. Belo Horizonte: FEP-MVZ, 1998. 221 p.

SANTOS, N. R. Comportamento sexual de touros Zebu (Bos taurus indicus) a pasto. (Tese de Doutorado). Belo Horizonte: Escola de Veterinária, UFMG, 2001.88p.

SANTOS, M. D.; TORRES, C. A. A.; RUAS, J. R. M. et al. Teste da libido e atividade de monta em touros da raça Nelore. Arq. Bras. Med. Vet. Zootec., v. 56, n. 4, p. 504-510, 2004.

SARREIRO, L. C. Estimativas de herdabilidade e correlações genéticas entre o perímetro escrotal, características seminais e libido de touros Nelore. (Dissertação de Mestrado). Belo Horizonte: Escola de Veterinária, UFMG, 2001.36 p.

SAS. User's Guide. SAS Inst., Inc., Cary, NC, 1996.

VALE FILHO, V. R.; BERGMANN, J. A. G.; ANDRADE, V. J. et al. Classificação andrológica por pontos (CAP), versus libido, na eficiência na fecundação de touros Nelore de 2 e 3 anos de idade, usados em estação de monta bem definida. In: ENCONTRO DE PESQUISA DA ESCOLA DE VETERINÁRIA DA UFMG, 14., v. 1, 1994, Belo Horizonte. Anais... Belo Horizonte: UFMG, 1994. p. 88. 